\title{
Sars-CoV-2 (COVID-19) Outbreak and Breast Cancer Surgery in Turkey
}

\author{
Güldeniz Karadeniz Çakmak ${ }^{1}$ (1), Vahit Özmen ${ }^{2}$ (1) \\ ${ }^{1}$ Zonguldak Bülent Ecevit University School of Medicine, Zonguldak, Turkey \\ ${ }^{2}$ İstanbul University İstanbul Faculty of Medicine, İstanbul, Turkey
}

Cite this article as: Karadeniz Çakmak G, Özmen V. Sars-CoV-2 (COVID-19) Outbreak and Breast Cancer Surgery in Turkey. Eur J Breast Health 2020; 16(2): 83-85.

The initial COVID-19 outbreak in Wuhan, China, in December 2019 had devastating global effects in a short period of time. The disease rapidly spread globally and was announced as a pandemic on March $11^{\text {th }}$ by World Health Organization when the first case was officially declared in Turkey. Turkish Ministry of Health has established COVID-19 Scientific Committee, and this took precautions on time and emphasized to accelerate public awareness in terms of social distancing, route of transmission, the period of infectivity and basic protective measures by means of social and mass media. Before the first official case the leaderships of all hospitals around the country, irrelevant of governmental or private origin, established COVID-19 committees and began to get prepared for the worst possible scenario about infection. All the medical staff and health care providers obliged to enter courses about personal protective measures. The shifts of all working groups re-organized in case of overloading.

Ministry of Health published a circular informing all physicians to discontinue every intervention, but only emergency and cancer surgeries where long-term outcomes are dependent on timely interventions. During this pandemic, one of the major issues is to protect health care providers from getting infected. To conserve or restore critical resources, including an intensive care unit, in-patient, and out-patient hospital beds, mechanical ventilators, transfusion products, and protective equipment, is vital for protecting uninfected patients and staff from extra viral exposure and intra-hospital transmission. The overwhelming number of infected patients with rapidly generated tragic health emergencies due to COVID-19 led to take strict precautions in the management of resources in terms of health care providers and protective equipment. Since COVID-19 pandemic became one of the greatest threatens against all humanity, causing thousands of deaths, the surgical algorithms have been impacted worldwide. As health care providers, we are currently facing a great challenge to provide assistance to all infected patients and, at the same time, treat non-deferrable oncologic and emergency cases. Not all cancer patients can be outlined, accordingly, we should be aware of the facts of our institute and focus on managing cancer with the maximum diligence during the pandemic. In this regard, to cancel the performance of elective surgical procedures is a must at this point. The estimation of expert projections about the full impact of COVID-19 varies and depends on multiple factors, one of the most important one is the optimal practice of social distancing which is a dynamic process and despite the vital importance in the disease course, this rule is not strictly obeyed in certain places. The pandemic is threatening our nation and all nations in all continents, and we should all be prepared to a potential increase in COVID-19 cases. Accordingly, various international surgical societies reported recommendations about possible strategies for the reorganization and rescheduling of surgical routine practice during this unpredictable period of time.

This editorial highlights the national and international approach to surgery under exceptional circumstances and challenges of breast surgery during COVID-19 pandemic. The importance of national governmental approach, the institutional organization, structure of triage of elective surgical interventions, physical and psychological preparations, infrastructure requirement analysis, the agility of mind, rational medical judgment and adjudication is also emphasized. This document could be a valid material to be used in routine clinical practice and potentially may serve as a cornerstone for advanced discussion on international similarities or differences about surgical approach algorithms under the shadow of COVID-19 pandemic which may provide beneficial recommendations for particularly breast surgeons in the state of emergency. Globally, we all share the same vision and mission for continuing to serve human well-being in the face of ambiguity and unpredictability. To establish our common goal we must accurately delineate priorities. With the key goals to reduce morbidity and mortality, minimize disease transmission, protect healthcare personnel and preserve healthcare system functioning, Cen- 
ters for Disease Control (CDC) and Prevention in the US suggests that "elective surgeries" at in-patient facilities be re-scheduled if possible and elective urgent inpatient diagnostic and surgical procedures are shifted to outpatient settings, when feasible (1). Similarly, The American College of Surgeons (ACS) has published guidance advising hospitals to discontinue elective surgery with guidance on the triage of non-emergent surgical procedures during the pandemic $(2,3)$. ACS also encourages to create a surgical review committee for COVID19-related surgical triage decision-making and recommends those decisions on surgery cases be made on a daily basis, no later than the day before surgery, by a leadership team representing surgery, anesthesiology, and nursing (4). Moreover, in conjunction with the surgical specialty societies ACS has launched triage criteria to serve as a resource for decision making and announced additional advice about triage of patients for cancer surgery $(5,6)$. The guiding principles for cancer care triage include resource considerations, cancer care coordination and general comments regarding cancer care triage by pandemic phases and institutional resources. These comments stresses to organize decision-making into three phases of the pandemic that describe the acuity of the local COVID-19 situation. Phase I; the semi-urgent setting, in which there are few COVID 19 patients, hospital resources are not exhausted, the institution still has intensive care unit ventilator capacity, and COVID trajectory not in rapid escalation phase. Phase II is defined as an urgent setting and there are many COVID 19 patients, intensive care unit and ventilator capacity limited, operation room supplies limited or COVID trajectory within the hospital in the rapidly escalating phase. Phase III is the phase when all hospital resources are routed to COVID 19 patients, no ventilator or intensive care unit capacity and operation room supplies exhausted. In regard to this phasing ACS's recommendations for each cancer type in terms of surgical management differs. ACS's COVID-19 Guideline for Triage of Breast Cancer Patients recommends surgery restricted to patients likely to have survivorship compromised if surgery not performed within the next 3 months during phase I. These cases that need to be done are as follows; patients completing neoadjuvant treatment, clinical stage T2 or N1 ER+/PR+/HER2 negative tumors, triple-negative or HER2 positive patients, discordant biopsies likely to be malignant and excision of malignant recurrence. Depending on the institutional resources, decisions may be made to proceed with surgery versus subjecting a patient to an immunocompromised state with neoadjuvant chemotherapy. ACS encourages breast conserving surgery whenever possible but defers definitive mastectomy and/or reconstruction (autologous and implant) until after the COVID 19 pandemic resolves. During phase II (urgent setting) and phase III, due to large numbers of COVID-19 patients and limited hospital resources surgery restricted to patients likely to have survivorship compromised if surgery not performed within the next few days or hours including, incision and drainage of breast abscess, evacuation of a hematoma, revision of an ischemic mastectomy flap and revascularization/revision of an autologous tissue flap. Under these circumstances, all other cases advised to be deferred (7). Recommendations for Prioritization, Treatment, and Triage of Breast Cancer Patients During the COVID-19 Pandemic developed by the COVID 19 Pandemic Breast Cancer The consortium which is made up of representatives from the National Accreditation Program for Breast Centers (NAPBC), Commission on Cancer (CoC) American Society for Breast Surgeons, ASBrS, and National Comprehensive Cancer Network (NCCN) $(8,9)$. The consortium comprises recommendations in regard to priority categories based on patient condition. Priority A defines a patient's condition as immediately life-threatening and clinically unsta- beyond 6-8 weeks could potentially impact the overall outcome and Priority $\mathrm{C}$ defines patient's condition as stable enough that services can be delayed for the duration of the COVID-19 pandemic. Recommendations to defer or perform surgery are similar to ACS's COVID-19 Guidelines for Triage of Breast Cancer Patients that phases I,II,III in ACS's guideline comprises priority categories of A,B,C in Consortium guideline (8). Society of Surgical Oncology (SSO) publicized a resource for management options of breast cancer during COVID-19 on $23^{\text {rd }}$ of March supporting the need for treatment decisions to be made on a case-by-case basis pending hospital resources and restrictions. SSO's recommends deferring prophylactic and risk-reducing surgery-reconstruction and surgery for atypia and benign breast disease at least 3 months. For ER+ DCIS, SSO' recommendation is to defer surgery for 3-5 months and treat with endocrine therapy and monitor monthly for progression. Untreated DCIS has high priority for surgery as soon as safe operation rooms are available. SSO encourages endocrine or chemotherapy in neoadjuvant fashion per multidisciplinary tumor board for ER+ Stage I-III invasive breast cancer. Patients with triple-negative/HER2+, T2N0-3M0 or T0-4N1-3M0 invasive breast cancer should begin neoadjuvant chemotherapy. Patients with T1N0M0 disease should be considered a high priority for surgery. For post-neoadjuvant chemotherapy setting patients with $\mathrm{ER}+$ invasive breast cancer with a partial/complete clinical response, converting to endocrine therapy could be considered in order to delay surgery versus surgery within 4-8 weeks. If the patient is also HER2+, converting to endocrine therapy could be considered in addition to anti-HER2 therapy in order to delay surgery. After neoadjuvant chemotherapy for triple-negative/HER2+ invasive breast cancer patients, surgery should only be delayed within a 4-8 week post-chemotherapy window, depending on the response as long as possible. These patients should be high priority for operation when deemed safe by the individual health system/hospital (10). Additionally, SSO strongly discourages not delaying patients with progressive disease on systemic therapy, angiosarcoma, and malignant phyllodes tumors and these cases should be considered for urgent surgery (10). The European Society of Surgical Oncology (ESSO) statement on COVID-19 advises no surgery for benign disease or risk-reduction should be performed (11). ESSO encourages maintaining weekly multidisciplinary team or tumor board meetings, preferably done remotely via video conferencing or telephone. The Surgical Royal Colleges of the United Kingdom and Ireland published guidance for surgeons working during the COVID-19 pandemic informing that the surgical workforce will need to adapt during the COVID-19 pandemic and listed the priorities in terms of the importance which are to maintain emergency surgery capabilities, to protect and preserve the surgical workforce, to fulfill alternate surgical roles and to fulfill alternate non-surgical roles (12). The overarching principles are to triage and deliver healthcare to patients for maximal benefit as in a mass casualty scenario and to protect and preserve the surgical workforce. This guideline defines acute patients as surgeons' priority and recommends any patient currently prioritized to undergo urgent planned surgery must be assessed for COVID-19 and the current greater risks of adverse outcomes factored into planning and consent (13). American Society of Clinical Oncology (ASCO) commented on CDC's guidance for health care facilities and ASC guidelines to answer the question Can/should surgery be canceled or delayed? If surgery is delayed, should patients be started earlier on neoadjuvant therapy if that is an available option? ASCO advice for clinicians and patients is to make individual determinations based on the potential harms of delaying needed cancer-related surgery; in many cases, these surgeries cannot be considered "elective" (14). ASCO also 
encourages to consider neoadjuvant therapy in some situations including early-stage breast cancer when available, but not in routine fashion. To consider neoadjuvant therapy may be reasonable to simply delay surgery in selected cases. ASCO advises to consider the risks of tumor progression with delay in definitive surgery should be weighed against the potential added burden on hospital resources, case complexity and patient risk of exposure to COVID-19 and emphasizes the issues of neoadjuvant therapy itself that should be also considered. These are the requirement of clinic visits and clinician-patient contact and immunosuppression caused by neoadjuvant treatment that is associated with risks to the patient.

Nationally, Turkey announced very strict regulations officially in terms of resource management on time. Elective procedures postponed immediately aiming to reduce hospital admissions and decrease transmission of disease between symptomatic and asymptomatic patients and health care staff. Moreover, this strategy serves to save resources including hospital beds, personal protective equipment, and preserve the surgical staff from getting infected. In Turkey, surgeons are all very well aware of the fact that our major mission is to preserve human resources and be prepared for the worst scenario ever. On March $29^{\text {th }}$, the total number of test performed in Turkey is 65.446, the official number of COVID-19 cases is 9217 and we lost 131 patients since the first diagnosis. Fortunately, Turkey has more than 20.000 adult intensive care beds all around the country most of which are ready to serve COVID patients, if required.

Our tumor boards declared that the management of new and old breast cancer patients during the COVID-19 period should be personalized according to hospital resources and restrictions. In all patients with DCIS, surgery may be deferred for 3-6 months together with monthly physical examination and ultrasound, ER+ patients will receive endocrine therapy in this period. Patients with triple-negative/ HER2+, T2N0-3M0 or T0-4N1-3M0 invasive breast cancer should begin neoadjuvant chemotherapy. We also recommend endocrine or chemotherapy in neo-adjuvant fashion for ER+ Stage I-III invasive breast cancer. After neoadjuvant chemotherapy for triple-negative/ HER2 + invasive breast cancer patients, surgery should be one of our priorities whenever the health system allows. Surgery will be delayed for 4-8 weeks if there is a good response to neoadjuvant chemotherapy. Hormonal therapy should be the first choice in old patients with ER+ breast cancer. And, patients with progressive disease on chemotherapy, malignant phyllodes, and aggressive sarcomas should have priority for surgery.

In regard to breast cancer surgery today in Turkey, high volume centers in which routine monthly volume is between 20 to 50 breast cancer cases, decreased the surgical interventions to the least possible rates, with great attention to patient and tumor biology and a shared decision making due to multidisciplinary tumor boards designed in a webbased fashion. In specific, only 17 patients $(17 / 48,35 \%)$ in the Breast Unit in the Istanbul Breast Center and 12 (12/32, 37.5\%) patients in the Breast Unit in Zonguldak Bulent Ecevit University School of
Medicine, who had decisions for surgery at diagnosis or completed neoadjuvant chemotherapy and within the last period of window period underwent surgery in the last month. The decision-making process is organized by means of periodic web-based multidisciplinary tumor boards and the patients are informed via phone calls. We encourage endocrine or chemotherapy in neoadjuvant fashion according to the decision in multidisciplinary tumor board for ER+ Stage I-III invasive breast cancer. Neoadjuvant chemotherapy is the preferred treatment of choice for patients with triple-negative/HER2+ or locally advanced disease, as well. However, in cases where surgery needs to be prioritized, to inform patients about the risks and benefits surgery under the circumstances of the pandemic is of paramount importance.

COVID-19 creates overwhelming challenges not only in the era of surgery but in all aspects of our healthcare systems. This is a dynamic process and evolving rapidly. Strategic planning during the COVID-19 pandemic will keep all health care providers safe and resistant which in turn allows us to provide the best quality of care to the nations we serve. This is a global war against an invisible enemy, we had to be tougher, stronger and together more than ever. Change has come to healthcare systems and change is coming to surgery at all. Nothing will be the same again; we have to adapt the provision of surgery. We, as scientists and physicians should act in a way to share experiences and recommendations in order to establish the best practices and save lives.

\section{References}

1. Available from: https://www.cdc.gov/coronavirus/2019-ncov/healthcarefacilities/guidance-hcf.html

2. Available from:https://www.facs.org/covid-19/clinical-guidance/electivecase

3. Available from:https://www.cms.gov/files/document/31820-cms-adultelective-surgery-and-procedures-recommendations.pdf

4. Available from:https://www.facs.org/covid-19/clinical-guidance/reviewcommittee

5. Available from:https://www.facs.org/covid-19/clinical-guidance/triage

6. Available from:https://www.facs.org/covid-19/clinical-guidance/electivecase/cancer-surgery

7. Available from:https://www.facs.org/covid-19/clinical-guidance/electivecase/breast-cancer

8. Available from:https://www.breastsurgeons.org/docs/news/The_COVID-19_Pandemic_Breast_Cancer_Consortium_Recommendations_EXECUTIVE_SUMMARY.pdf

9. Ontario Health, Cancer Care Ontario, "Pandemic Planning Clinical Guideline for Patients with Cancer”. Available from: https://www.accccancer.org/docs/document/cancer-program-fundamentals/oh-ccopandemic-planning-clinical-guidelines (accessed March 23, 2020)

10. Available from: https://www.surgonc.org/wp-content/uploads/2020/03/ Breast-Resource-during-COVID-19-3.23.20.pdf

11. Available from: https://www.essoweb.org/news/esso-statement-covid-19/

12. Available from: https://www.rcseng.ac.uk/coronavirus/joint-guidancefor-surgeons-v1/

13. Available from: https://www.rcseng.ac.uk/coronavirus/joint-guidancefor-surgeons-v2/

14. Available from: https://www.asco.org/asco-coronavirus-information/careindividuals-cancer-during-covid-19 